\title{
Improved measurement of the lifetime of the $\tau$ lepton
}

\section{OPAL Collaboration}

G. Alexander ${ }^{\mathrm{w}}$, J. Allison ${ }^{\mathrm{p}}$, N. Altekamp ${ }^{\mathrm{e}}$, K. Ametewee ${ }^{\mathrm{y}}$, K.J. Anderson ${ }^{\mathrm{i}}$, S. Anderson ${ }^{\ell}$, S. Arcelli ${ }^{\text {b }}$, S. Asai ${ }^{x}$, D. Axen ${ }^{\text {ac }}$, G. Azuelos ${ }^{\text {r.l }}$, A.H. Ball ${ }^{q}$, E. Barberio ${ }^{z}$, R.J. Barlow ${ }^{\text {p }}$, R. Bartoldus ${ }^{\text {c }}$, J.R. Batley ${ }^{\mathrm{e}}$, G. Beaudoin ${ }^{\mathrm{r}}$, J. Bechtluft ${ }^{\mathrm{n}}$, C. Beeston ${ }^{\mathrm{p}}$, T. Behnke ${ }^{\mathrm{h}}$, A.N. Bell ${ }^{\mathrm{a}}$, K.W. Bell ${ }^{\mathrm{t}}$, G. Bella ${ }^{\mathrm{w}}$, S. Bentvelsen ${ }^{\mathrm{h}}$, P. Berlich ${ }^{\mathrm{j}}$, S. Bethke ${ }^{\mathrm{n}}$, O. Biebel ${ }^{\mathrm{n}}$, V. Blobel ${ }^{\text {h }}$, I.J. Bloodworth ${ }^{\mathrm{a}}$, J.E. Bloomer ${ }^{\mathrm{a}}$, P. Bock ${ }^{\mathrm{k}}$, H.M. Bosch ${ }^{\mathrm{k}}$, M. Boutemeur ${ }^{\mathrm{r}}$, B.T. Bouwens ${ }^{\ell}$, S. Braibant ${ }^{\ell}$, P. Bright-Thomas ${ }^{y}$, R.M. Brown ${ }^{t}$, H.J. Burckhart ${ }^{\text {h }}$, C. Burgard ${ }^{\text {aa }}$, R. Bürgin ${ }^{j}$, P. Capiluppi ${ }^{\text {b }}$, R.K. Carnegie ${ }^{f}$, A.A. Carter ${ }^{\mathrm{m}}$, J.R. Carter ${ }^{\mathrm{e}}$, C.Y. Chang ${ }^{\mathrm{a}}$, C. Charlesworth ${ }^{\mathrm{f}}$, D.G. Charlton ${ }^{\mathrm{a}, 2}$, D. Chrisman ${ }^{\mathrm{d}}$, S.L. Chu ${ }^{\mathrm{d}}$, P.E.L. Clarke ${ }^{o}$, I. Cohen ${ }^{w}$, J.E. Conboy ${ }^{o}$, O.C. Cooke $^{\text {p }}$, M. Cuffiani ${ }^{b}$, S. Dado ${ }^{v}$, C. Dallapiccola ${ }^{q}$, G.M. Dallavalle ${ }^{b}$, C. Darling ${ }^{\text {ae }}$, S. De Jong ${ }^{\ell}$, L.A. del Pozo ${ }^{\text {h }}$, M.S. Dixit ${ }^{g}$, E. do Couto e Silva ${ }^{\ell}$, M. Doucet ${ }^{r}$, E. Duchovni ${ }^{z}$, G. Duckeck ${ }^{\text {h }}$, I.P. Duerdoth ${ }^{\mathrm{p}}$, J.E.G. Edwards ${ }^{\mathrm{p}}$, P.G. Estabrooks ${ }^{\mathrm{f}}$, H.G. Evans ${ }^{\mathrm{i}}$, M. Evans ${ }^{\mathrm{m}}$, F. Fabbri ${ }^{\mathrm{b}}$, P. Fath ${ }^{k}$, F. Fiedler ${ }^{\ell}$, M. Fierro ${ }^{\text {b }}$, H.M. Fischer ${ }^{\mathrm{c}}$, R. Folman ${ }^{\mathrm{z}}$, D.G. Fong ${ }^{\mathrm{q}}$, M. Foucher ${ }^{\mathrm{q}}$, H. Fukui ${ }^{x}$, A. Fürtjes ${ }^{\text {h }}$, P. Gagnon ${ }^{g}$, A. Gaidot ${ }^{\text {u }}$, J.W. Gary ${ }^{\text {d, J. Gascon }}{ }^{\text {r }}$,

S.M. Gascon-Shotkin ${ }^{q}$, N.I. Geddes ${ }^{t}$, C. Geich-Gimbel ${ }^{\text {c }}$, S.W. Gensler ${ }^{i}$, F.X. Gentit ${ }^{u}$, T. Geralis ${ }^{\mathrm{t}}$, G. Giacomelli ${ }^{\mathrm{b}}$, P. Giacomelli ${ }^{\mathrm{d}}$, R. Giacomelli ${ }^{\mathrm{b}}$, V. Gibson ${ }^{\mathrm{e}}$, W.R. Gibson ${ }^{\mathrm{m}}$, D.M. Gingrich ${ }^{\text {ad, },}$, J. Goldberg ${ }^{\text {v }}$, M.J. Goodrick ${ }^{\mathrm{e}}$, W. Gorn ${ }^{\mathrm{d}}$, C. Grandi ${ }^{\mathrm{b}}$, E. Gross ${ }^{z}$, M. Gruwé ${ }^{\mathrm{h}}$, C. Hajdu ${ }^{\text {af }}$, G.G. Hanson ${ }^{\ell}$, M. Hansroul ${ }^{\mathrm{h}}$, M. Hapke ${ }^{\mathrm{m}}$, C.K. Hargrove ${ }^{\mathrm{g}}$, P.A. Hart ${ }^{i}$, C. Hartmann ${ }^{c}$, M. Hauschild ${ }^{\text {h }}$, C.M. Hawkes ${ }^{\text {e }}$, R. Hawking ${ }^{\text {h }}$, R.J. Hemingway ${ }^{f}$, G. Herten ${ }^{\text {i }}$, R.D. Heuer ${ }^{\text {h }}$, M.D. Hildreth ${ }^{\text {h }}$, J.C. Hill ${ }^{\mathrm{e}}$, S.J. Hillier ${ }^{\mathrm{a}}$, T. Hilse ${ }^{\mathrm{j}}$, P.R. Hobson ${ }^{y}$, R.J. Homer ${ }^{a}$, A.K. Honma ab,1, D. Horváth ${ }^{\text {af, } 3}$, R. Howard ${ }^{\text {ac }}$, R.E. Hughes-Jones ${ }^{p}$,

D.E. Hutchcroft ${ }^{\mathrm{e}}$, P. Igo-Kemenes ${ }^{k}$, D.C. Imrie ${ }^{y}$, M.R. Ingram ${ }^{p}$, A. Jawahery ${ }^{\mathrm{q}}$, P.W. Jeffreys ${ }^{\mathrm{t}}$, H. Jeremie ${ }^{\mathrm{r}}$, M. Jimack ${ }^{\mathrm{a}}$, A. Joly ${ }^{\mathrm{r}}$, G. Jones ${ }^{\mathrm{p}}$, M. Jones ${ }^{\mathrm{f}}$, R.W.L. Jones ${ }^{\mathrm{h}}$, U. Jost ${ }^{\text {k }}$, P. Jovanovic ${ }^{\text {a }}$, J. Kanzaki ${ }^{x}$, D. Karlen ${ }^{\text {f }}$, T. Kawamoto ${ }^{x}$, R.K. Keeler ${ }^{\text {ab }}$, R.G. Kellogg ${ }^{\mathrm{q}}$, B.W. Kennedy ${ }^{\mathrm{t}}$, J. King ${ }^{\mathrm{m}}$, J. Kirk ${ }^{\text {ac }}$, S. Kluth ${ }^{\mathrm{h}}$, T. Kobayashi ${ }^{\mathrm{x}}$, M. Kobel ${ }^{\mathrm{j}}$, D.S. Koetke ${ }^{\text {f }}$, T.P. Kokott ${ }^{c}$, S. Komamiya ${ }^{x}$, R. Kowalewski ${ }^{\text {h }}$, T. Kress ${ }^{k}$, P. Krieger ${ }^{f}$, J. von Krogh ${ }^{k}$, P. Kyberd ${ }^{m}$, G.D. Lafferty ${ }^{p}$, H. Lafoux ${ }^{u}$, R. Lahmann ${ }^{q}$, W.P. Lai ${ }^{\text {s, }}$ D. Lanske ${ }^{\text {n }}$, J. Lauber ${ }^{\circ}$, J.G. Layter ${ }^{\text {d }}$, A.M. Lee ${ }^{\text {ae }}$, E. Lefebvre $^{r}$, D. Lellouch ${ }^{\text {}}$, J. Letts ${ }^{\text {b }}$, L. Levinson ${ }^{z}$, C. Lewis ${ }^{o}$, S.L. Lloyd ${ }^{m}$, F.K. Loebinger ${ }^{p}$, G.D. Long ${ }^{q}$, B. Lorazo ${ }^{r}$, M.J. Losty ${ }^{g}$, J. Ludwig ${ }^{j}$, A. Luig ${ }^{j}$, A. Malik ${ }^{\mathrm{u}}$, M. Mannelli ${ }^{\mathrm{h}}$, S. Marcellini ${ }^{\mathrm{b}}$, C. Markus ${ }^{\mathrm{c}}$, 
A.J. Martin ${ }^{\mathrm{m}}$, J.P. Martin ${ }^{\mathrm{r}}$, G. Martinez ${ }^{\mathrm{q}}$, T. Mashimo ${ }^{\mathrm{x}}$, W. Matthews ${ }^{\mathrm{y}}$, P. Mättig ${ }^{\mathrm{c}}$, W.J. McDonald ${ }^{\text {ad }}$, J. McKenna ${ }^{\text {ac }}$, E.A. Mckigney ${ }^{\text {, }}$ T.J. McMahon ${ }^{\text {a }}$, A.I. McNab ${ }^{\mathrm{m}}$, F. Meijers ${ }^{h}$, S. Menke ${ }^{c}$, F.S. Merritt ${ }^{i}$, H. Mes ${ }^{g}$, J. Meyer ${ }^{\text {aa }}$, A. Michelini ${ }^{\text {h }}$, G. Mikenberg ${ }^{z}$, D.J. Miller ${ }^{\circ}$, R. Mir ${ }^{2}$, W. Mohr ${ }^{\text {j, A. Montanari }}{ }^{\text {, }}$, T. Mori ${ }^{x}$, M. Morii ${ }^{x}$, U. Müller ${ }^{c}$, B. Nellen ${ }^{c}$, B. Nijihar ${ }^{\mathrm{p}}$, R. Nisius ${ }^{\mathrm{h}}$, S.W. O'Neale ${ }^{\mathrm{a}}$, F.G. Oakham ${ }^{\mathrm{g}}$, F. Odorici ${ }^{\mathrm{b}}$, H.O. Ogren ${ }^{\ell}$, T. Omori ${ }^{x}$, M.J. Oreglia ${ }^{\mathrm{i}}$, S. Orito ${ }^{x}$, M. Palazzo ${ }^{\text {, }}$, J. Pálinkás ${ }^{\mathrm{ag}, 4}$, J.P. Pansart ${ }^{\mathrm{u}}$, G. Pásztor ${ }^{\text {af }}$, J.R. Pater ${ }^{\mathrm{p}}$, G.N. Patrick ${ }^{\mathrm{t}}$, M.J. Pearce ${ }^{\mathrm{a}}$, S. Petzold ${ }^{\mathrm{aa}}$, J.E. Pilcher ${ }^{i}$, J. Pinfold ${ }^{\text {ad }}$, D.E. Plane ${ }^{h}$, P. Poffenberger ${ }^{a b}$, B. Poli ${ }^{b}$, A. Posthaus ${ }^{c}$, H. Przysiezniak ${ }^{\text {ad }}$, D.L. Rees ${ }^{a}$, D. Rigby ${ }^{a}$, M.G. Rison ${ }^{\mathrm{e}}$, S.A. Robins ${ }^{\mathrm{m}}$, N. Rodning ${ }^{\text {ad }}$, J.M. Roney ${ }^{\text {ab }}$, A. Rooke ${ }^{\circ}$, E. Ros ${ }^{\text {h }}$, A.M. Rossi ${ }^{b}$, M. Rosvick ${ }^{\text {ab }}$, P. Routenburg ${ }^{\text {ad }}$, Y. Rozen ${ }^{\text {h }}$, K. Runge ${ }^{j}$, O. Runolfsson ${ }^{\text {h }}$, D.R. Rust ${ }^{\ell}$, R. Rylko ${ }^{y}$, E.K.G. Sarkisyan ${ }^{w}$, M. Sasaki ${ }^{\text {, C. Sbarra }}{ }^{\text {b }}$, A.D. Schaile ${ }^{\text {h,5 }}$, O. Schaile ${ }^{\text {j }}$, F. Scharf ${ }^{\text {c }}$, P. Scharff-Hansen ${ }^{\text {h }}$, P. Schenk ${ }^{d}$, B. Schmitt ${ }^{\text {, }}$, M. Schröder ${ }^{\text {h }}$, H.C. Schultz-Coulon ${ }^{j}$, M. Schulz ${ }^{\text {h }}$, P. Schütz ${ }^{\text {, }}$, J. Schwiening ${ }^{c}$, W.G. Scott ${ }^{t}$, T.G. Shears ${ }^{p}$, B.C. Shen ${ }^{d}$, C.H. Shepherd-Themistocleous ${ }^{\text {aa }}$, P. Sherwood ${ }^{\text {, }}$, G.P. Siroli ${ }^{\mathrm{b}}$, A. Sittler ${ }^{\text {aa }}$, A. Skillman ${ }^{\circ}$, A. Skuja ${ }^{\text {q }}$, A.M. Smith ${ }^{\mathrm{h}}$, T.J. Smith ${ }^{\text {ab }}$, G.A. Snow ${ }^{q}$, R. Sobie ${ }^{\text {ab }}$, S. Söldner-Rembold ${ }^{j}$, R.W. Springer ${ }^{\text {ad }}$, M. Sproston ${ }^{t}$, A. Stahl ${ }^{\text {c }}$, M. Starks ${ }^{\ell}$, K. Stephens ${ }^{\text {P }}$, J. Steuerer ${ }^{\text {aa }}$, B. Stockhausen ${ }^{\text {, }}$, D. Strom ${ }^{\text {s }}$, F. Strumia ${ }^{\text {h }}$, P. Szymanski ${ }^{t}$, R. Tafirout ${ }^{r}$, H. Takeda ${ }^{x}$, P. Taras ${ }^{r}$, S. Tarem ${ }^{\text {v }}$, M. Tecchio ${ }^{h}$, N. Tesch ${ }^{c}$, M. Thiergen ${ }^{j}$, M.A. Thomson ${ }^{h}$, E. von Törne ${ }^{c}, S$. Towers ${ }^{f}$, M. Tscheulin ${ }^{j}$, E. Tsur ${ }^{\text {w }}$, A.S. Turcot ${ }^{i}$, M.F. Turner-Watson ${ }^{h}$, P. Utzat ${ }^{k}$, R. Van Kooten ${ }^{\ell}$, G. Vasseur ${ }^{\mathrm{u}}$, M. Verzocchi ${ }^{\mathrm{j}}$, P. Vikas ${ }^{\mathrm{r}}$, M. Vincter ${ }^{\mathrm{ab}}$, E.H. Vokurka ${ }^{\mathrm{p}}$, F. Wäckerle ${ }^{\mathrm{j}}$, A. Wagner ${ }^{\text {aa }}$, C.P. Ward ${ }^{\text {e }}$, D.R. Ward ${ }^{\text {e }}$, J.J. Ward ${ }^{\text {}}$, P.M. Watkins ${ }^{\text {a }}$, A.T. Watson ${ }^{\text {a }}$, N.K. Watson $^{g}$, P. Weber ${ }^{f}$, P.S. Wells ${ }^{h}$, N. Wermes ${ }^{c}$, J.S. White ${ }^{a b}$, B. Wilkens ${ }^{j}$, G.W. Wilson ${ }^{a}$, J.A. Wilson ${ }^{a}$, T. Wlodek ${ }^{z}$, G. Wolf ${ }^{z}$, S. Wotton ${ }^{k}$, T.R. Wyatt ${ }^{p}$, S. Xella ${ }^{b}$,

S. Yamashita ${ }^{x}$, G. Yekutieli ${ }^{z}$, K. Yoshimura ${ }^{x}$, V. Zacek ${ }^{r}$

a School of Physics and Space Research. University of Birmingham, Birmingham B15 2TT, UK

' Dipartimento di Fisica dell' Università di Bologna and INFN, I-40126 Bologna, Italy

'Physikalisches Institut, Universität Bonn, D-53115 Bonn, Germany

'Department of Physics, University of California, Riverside, CA 92521, USA

- Cavendish Laboratory, Cambridge CB3 OHE, UK

'Onawa-Carieton Institute for Physics, Department of Physics, Carleton University, Ottawa, Ont. KIS 5B6. Canada

\& Centre for Research in Particle Physics, Carleton University. Ottawa, Ont. KIS 5B6, Canada

${ }^{\text {h }}$ CERN, European Organisation for Particle Physics, CH-121/ Geneva 23, Switzerland

i Enrico Fermi Institute and Department of Physics, University of Chicago, Chicago, IL 60637, USA

i Fakultät für Physik. Albert Ludwigs Universität, D-79104 Freiburg, Germany

k Physikalisches Institut. Universität Heidelberg, D.69120 Heidelberg, Germany

'Indiana University, Department of Physics, Swain Hall West 117, Bloomington, IN 47405, USA

${ }^{m}$ Queen Mary and Westfield College. University of London. London EI 4NS, UK

"Technische Hochschule Aachen, III Physikalisches Institul, Sommerfeldstrasse 26-28, D-52056 Aachen, Germany "University College London, London WCIE 6BT, UK

${ }^{p}$ Department of Physics, Schuster Laboratory, The University, Manchester M13 9PL, UK

${ }^{4}$ Department of Physics, University of Maryland, College Park, MD 20142, USA

'Laboratoire de Physique Nucléaire, Université de Montréal, Montréal, Que. H3C 3J7. Canada

$s$ University of Oregon, Department of Physics, Eugene, OR 97403. USA

1 Rutherford Appleton Laboratory, Chilton. Didcot, Oxfordshire OXII OQX, UK "CEA, DAPNIA/SPP, CE-Saclay. F-91191 Gif-sur-Yvette, France 


\author{
" Department of Physics, Technion-Israel Institute of Technology, Haifa 32000, Israel \\ " Department of Physics and Astronomy, Tel Aviv University. Tel Aviv 69978, Israel \\ ${ }^{x}$ International Centre for Elementary Particle Physics and Department of Physics, University of Tokyo, Tokyo 113, Japan \\ and Kobe University, Kobe 657, Japan \\ ${ }^{y}$ Brunel University, Uxbridge, Middlesex UB8 $3 P H, U K$ \\ "Particle Physics Department, Weizmann Institute of Science, Rehovot 76100, Israel \\ ail Universität Hamburg/DESY, II Institut für Experimental Physik. Notkestrasse 85. D-22607 Hamburg, Germany \\ ab University of Victoria, Department of Physics, P O Box 3055, Victoria, BC V8W 3P6, Canada \\ ac University of British Columbia, Department of Physics, Vancouver, BC V6T IZI, Canada \\ ad University of Alberta, Department of Physics, Edmonton, AB T6G 2JI, Canada \\ ae Duke University. Dept of Physics, Durham, NC 27708-0305, USA \\ al Research Institute for Particle and Nuclear Physics, H-1525 Budapest, P O Box 49, Hungary \\ ag Institute of Nuclear Research. H-400I Debrecen, $P$ O Box 5I, Hungary \\ Received 20 February 1996 \\ Editor: K. Winter
}

\begin{abstract}
A new measurement of the $\tau$ lifetime is presented. It uses data collected with the OPAL detector during 1994, which almost doubles the size of the OPAL $\tau$ sample. Two statistically independent techniques are used: an impact parameter analysis of one-prong decay tracks and a fit to the decay length distribution of three-prong decays. The lifetime obtained from the 1994 data by combining the results of these methods is $\tau_{\tau}=289.7 \pm 2.5$ (stat) \pm 1.5 (sys) fs. When combined with the previous OPAL $\tau$ lifetime measurement the improved $\tau$ lifetime is $\tau_{\tau}=289.2 \pm 1.7$ (stat.) \pm 1.2 (sys.) fs.
\end{abstract}

\section{Introduction}

We present a new measurement of the $\tau$ lifetime using data collected by OPAL in 1994. With the inclusion of this data, the OPAL $\tau$ data sample is almost doubled. The two statistically independent methods used to measure the lifetime are the same as those used in the previous analyses $[1,2]$ : the impact parameter method applied to $\tau$ decays to a single charged track and the decay length method applied to decays to three charged tracks.

The $\tau$ lifetime is one of the inputs needed to test $\tau-\mu$ charged-current lepton universality within the Standard Model, along with the $\tau$ mass and electronic branching ratio and the $\mu$ mass and lifetime. These quantities are related to the electroweak couplings, $g_{\ell}$, via

\footnotetext{
' and at TRIUMF, Vancouver, Canada V6T $2 \mathrm{~A} 3$.

2 and Royal Society University Research Fellow.

${ }^{3}$ and Institute of Nuclear Research, Debrecen, Hungary.

${ }^{4}$ and Depart of Experimental Physics, Lajos Kossuth University, Debrecen, Hungary.

s and Ludwig-Maximilians-Universität, München, Germany.
}

$$
\left(\frac{g_{\tau}}{g_{\mu}}\right)^{2}=\left(\frac{\tau_{\mu}}{\tau_{\tau}}\right)\left(\frac{m_{\mu}}{m_{\tau}}\right)^{5} \operatorname{BR}\left(\tau \rightarrow \mathrm{e} \bar{\nu}_{\mathrm{e}} \nu_{\tau}\right)\left(1-\delta_{r}\right),
$$

where $\delta_{r}$, the electroweak correction, is 0.0004 [3]. In recent years, the improved measurements of the $\tau$ lepton mass [4,5], as well as higher precision in the $\tau$ leptonic branching ratio and $\tau$ lifetime measurements [5], have led to a much improved test of lepton universality using this relation. Although current results are consistent with equal electroweak couplings for the $\tau$ and $\mu$, it remains important to continue to improve the precision of this fundamental comparison.

\section{The OPAL detector}

A complete description of the OPAL detector can be found elsewhere [6], and only the components relevant to this analysis, namely the central tracking detectors, are described here. The coordinate system used has $z$ along the electron direction and $x$ pointing towards the ring centre. The polar angle, $\theta$, is defined relative to the $+z$-axis and the azimuthal angle, $\phi$, is 
defined relative to the $+x$-axis. The radius, $r$, is the distance from the $z$-axis.

The OPAL central tracking system comprises drift chambers that measure the $z$ coordinate at its outer radius, within which are contained the jet chamber, the vertex drift chamber and the silicon microstrip vertex detector. The jet chamber provides up to 159 radial measurements of a track from its first layer at $25.5 \mathrm{~cm}$ to its outermost at $183.5 \mathrm{~cm}$. The vertex drift chamber provides 12 radial measurements from $10.3 \mathrm{~cm}$ to $16.2 \mathrm{~cm}$ and 6 stereo measurements from $18.8 \mathrm{~cm}$ to $21.3 \mathrm{~cm}$. The three drift chambers are contained in a 4-bar pressure vessel. The silicon microvertex detector [7], which surrounds the $5.3 \mathrm{~cm}$-radius berylliumcomposite beam pipe at the interaction point, provides two layers of readout in both $r-\phi$ and $z$ coordinates. Only the $r-\phi$ information is used in this analysis. The entire tracking system is contained in a solenoidal magnetic field of $0.435 \mathrm{~T}$. The two track resolution of the jet chamber and the vertex chamber is about $2 \mathrm{~mm}$, and results in high efficiency to reconstruct collimated 3-prong $\tau$ decays. The impact parameter resolution of high momentum tracks reconstructed in the drift chambers and containing silicon detector hits is about $18 \mu \mathrm{m}$ in $r-\phi$.

\section{Event selection}

Approximately $46 \mathrm{pb}^{-1}$ of data were collected in 1994 at a single energy, $\sqrt{s} \approx 91.16 \mathrm{GeV}$, near the $\mathrm{Z}^{0}$ peak. From these data, $\tau$ events were selected with approximately $75 \%$ efficiency by requiring two lowmultiplicity back-to-back jets in an event, yielding 51007 candidate $\tau$-pair events. Full details of this selection can be found in [8]. After this selection, a measurement of the beam position for the LEP fill from which the event comes was required, and events without a good position measurement are rejected.

For each event, the thrust axis is calculated using information from charged tracks. Then, each event is divided into two hemispheres by the plane perpendicular to the thrust axis and passing through the interaction point. In the impact parameter analysis, a hemisphere is used if it contains exactly one charged track (hence the term one-prong). In the decay length analysis, a hemisphere is used if it contains exactly three charged tracks (hence three-prong).
The final selections for the two analyses are slightly different. For the impact parameter measurement, the following cuts are made. To reduce contributions from $\mu$-pair and Bhabha events, the angle between the $x-y$ projections of the one-prong track momentum vector and the reflection through a line perpendicular to it of the sum of the momenta of the tracks in the opposite hemisphere must be greater than $2 \mathrm{mrad}$. This cut has a $96.5 \%$ efficiency for the $\tau$-pair event data and rejects $37.5 \%$ of $\mu$-pair events as determined in a study of $\mu$ pair Monte Carlo events. When the two $\tau$ candidates appear to decay into single charged particles having the same sign, the event is rejected. Finally, the tracks are required to have the following three properties: at least one silicon detector hit in the $r-\phi$ plane, at least half the geometrically-possible number of hits in the central jet chamber, and a track impact parameter error, including the beam size contribution, of less than $0.1 \mathrm{~cm}$. After all these cuts, there are 62885 tracks for the impact parameter analysis.

For the decay length analysis, the multihadronic background is reduced by rejecting events which pass the OPAL multihadron selection [8]. In addition, tracks from probable photon conversions and $K_{s}^{0}$ decays are rejected [2]. The number of three-prong decay candidates remaining is 11839 .

\section{LEP beam parameters}

For both the impact parameter and decay length measurements, the beam centroid position, measured using charged tracks in multihadron events as described in [2], is used as the $\tau$ production point. The uncertainty in the beam centroid position is $\left(\delta \bar{x}_{b}, \delta \bar{y}_{b}\right)=( \pm 19, \pm 7) \mu \mathrm{m}$. The beam size is also relevant, since for the decay length method the $\tau$ production point uncertainty, as approximated by the beam ellipse, contributes directly to the decay length uncertainty. In the one-prong measurement the beam size contributes directly to the impact parameter resolution. In 1994, the beam size measured by OPAL was $\left(\sigma_{x}, \sigma_{v}\right)=(124,10) \mu \mathrm{m}$ with uncertainties $\left(\delta \sigma_{x}, \delta \sigma_{y}\right)=( \pm 7, \pm 3) \mu \mathrm{m}$. These uncertainties include the statistical measurement errors on the beam size and the variation in the beam size due to emittance changes in a fill. 


\section{Monte Carlo simulation}

Samples of $600000 \tau$-pair and $\mu$-pair events each were generated using the KORALZ 4.0 event generator [9] and were processed through the full detector simulation [10]. The input lifetime in the $\tau$-pair sample was $295.6 \mathrm{fs}$ and the input beam size was $(127,12) \mu \mathrm{m}$ in $(x, y)$, consistent with the measured beam size. The $\tau$ Monte Carlo simulation is used in the impact parameter analysis to extract the lifetime through a direct comparison with the data.

The resolution on the impact parameter, $d_{0}$, the distance of closest approach in the $r-\phi$ plane of a track to the interaction point, is better in the Monte Carlo simulation than in the data. Therefore the $d_{0}$ of tracks in the Monte Carlo simulation is smeared. Candidate $\mu$-pair events were used to compare data and Monte Carlo simulation to determine smearing factors to make the resolutions agree. In previous years, it was necessary to degrade the Monte Carlo simulation resolution significantly in order to obtain agreement with the data. However, for 1994, the OPAL detector simulation has been greatly improved, and so the extra smearing factors needed are quite small.

The smearing factors were determined by comparing the $\Sigma d_{0}$ distribution for $\mu$-pairs between data and Monte Carlo simulation, where $\Sigma d_{0}$ is the miss distance in $r-\phi$ at the beam position between the two muon tracks in an event. The $\Sigma d_{0}$ distribution was used since it is independent of the beam size and position, allowing use of the full $\phi$ range. This distribution is well described by the superposition of two Gaussians. In the $\mu$-pair data, the relative fractions are $88 \%$ with a width of $26 \mu \mathrm{m}$ and $12 \%$ with a width of $47 \mu \mathrm{m}$. A double Gaussian smearing was used in the Monte Carlo simulation to match the resolutions; $95 \%$ of the tracks had their $d_{0}$ smeared by $6.5 \mu \mathrm{m}$, with the rest smeared by $30 \mu \mathrm{m}$. These smearing factors were applied in the $\tau$ Monte Carlo simulation used in the impact parameter analysis.

In the decay length analysis, the $\tau$ Monte Carlo events were used primarily to check for any bias in the method, for which the unsmeared Monte Carlo was sufficient.

\section{Backgrounds}

The background contributions in the one-prong sample were evaluated using samples of Bhabha, muon and two photon Monte Carlo simulation events. The level of background was determined using the methods described in [11]. The background remaining in the 1-prong sample, after all cuts, is predominantly due to $\mu$-pair events and is $1.21 \pm 0.29 \%$.

In the three-prong sample, the only significant background is from multihadronic events. This contribution has been evaluated by studying two million JETSET 7.4 Monte Carlo simulation events [12,13]. The background level in the Monte Carlo simulation was calibrated by a comparison of the charged track and electromagnetic cluster multiplicity distributions for data and Monte Carlo simulation, for events which passed the $\tau$-pair selection cuts except for the multiplicity cuts. The resulting background fraction was $0.58 \pm 0.20 \%$, where the error includes both statistical and systematic uncertainties.

\section{Lifetime measurement using the impact parameter method}

The impact parameter, $d_{0}$, is defined in Section $5 . \mathrm{A}$ signed impact parameter distribution is used to measure the $\tau$ lifetime using one-prong decays. The impact parameter of a track is given a positive sign when the track crosses the thrust axis in the same hemisphere in which it lies and is given a negative sign otherwise. This distribution is formed for both data and Monte Carlo simulation and the trimmed means of each distribution are calculated. The trimmed mean is defined as the mean of the distribution after equal percentages of the highest and lowest entries are removed. It is used instead of the mean to make the measurement less sensitive to the tails of the distribution. The relationship between the lifetime and the trimmed mean, $\bar{d}$, is obtained using the Monte Carlo simulation:

$\tau_{\text {data }}=\bar{d}_{\text {data }} \times \frac{\tau_{\mathrm{mc}}}{\bar{d}_{\mathrm{mc}}}$

A $10 \%$ trim is used, that is, the top and bottom $5 \%$ of entries are excluded.

Since the beam is elliptical, its contribution to the $d_{0}$ uncertainty of horizontal tracks will be smaller 


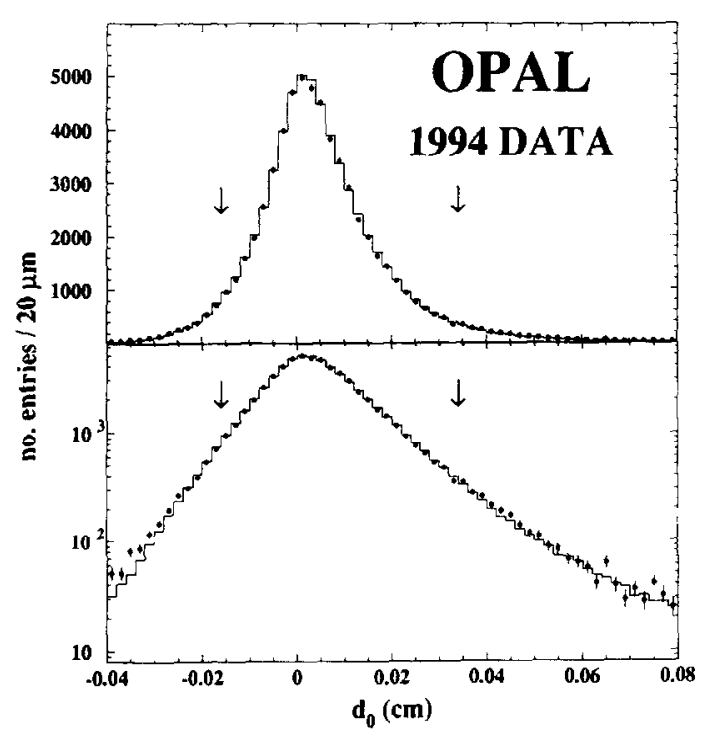

Fig. I. The $d_{0}$ distributions for the one-prong impact parameter analysis. The points with error bars show the distribution from the data, and the histogram is the result obtained from the $\tau$-pair Monte Carlo simulation, weighted to a lifetime value of $290 \mathrm{fs}$. The arrows indicate the $10 \%$ trim points.

than that of vertical tracks. Therefore, the trimmed mean is determined in six $\phi$ bins such that tracks which have approximately equal beam contributions to their uncertainties lie in the same bin. The $\phi$ range from 0 to $90^{\circ}$ is divided into six equal sections of $15^{\circ}$. Each $15^{\circ}$ division is reflected across the $x$-axis, and then both original and reflection are reflected across the $y$-axis, forming four separate sections. These four sections are combined to form one $\phi$ bin. The trimmed means of each $\phi$ bin are compared for data and Monte Carlo simulation and the six resulting lifetime measurements, which are consistent within statistical errors, are combined in a weighted average. The lifetime obtained from combining these bins is $287.5 \pm 3.5 \pm 1.1 \mathrm{fs}$, where the errors are the statistical uncertainties from data and Monte Carlo simulation respectively. The Monte Carlo simulation statistical error was included in the systematic errors, as discussed below.

The signed impact parameter distributions for data and Monte Carlo simulation for the six $\phi$ bins combined are shown in Fig. 1. The Monte Carlo simulation histogram has been re-weighted to a lifetime of $290 \mathrm{fs}$. The arrows represent the $10 \%$ trim points. There is good agreement between the two histograms: the $\chi^{2}$ between the two histograms over the 30 bins between the trim points is 26.8 , corresponding to a probability of $58 \%$.

The lifetime was evaluated for different ranges of the track polar angle and momentum, for different trim values, as a function of the number of tracks in the opposite hemisphere, and also using a thrust axis which includes electromagnetic calorimeter information. The lifetime values so obtained are consistent with the primary result.

A systematic error is assigned to rellect the different impact parameter resolutions observed in data and Monte Carlo simulation. The lifetime has been determined by using different smearing factors, and has been found to be stable even for smearing factors which give very poor agreement between the data and the Monte Carlo $\mu$-pair distributions. The systematic error assigned covers the range of lifetime variations obtained by using smearing prescriptions which give a probability for agreement between data and Monte Carlo simulation histograms of greater than $10 \%$.

The procedure used to calibrate the central detectors can result in possible misalignments between detectors. There is evidence for such misalignments since the impact parameter distribution for $\mu$-pairs is described by two Gaussians with means $10 \mu \mathrm{m}$ apart. The effect of this possible misalignment is checked by shifting the $d_{0}$ values of Monte Carlo simulation tracks by amounts varying from 5 to $40 \mu \mathrm{m}$. A linear variation in the lifetime is observed, and the systematic error is assigned by allowing for a $10 \mu \mathrm{m}$ shift in $d_{0}$.

Systematic errors are also assigned due to uncertainties in the beam size and position, which were described in Section 4. An increase in the beam size will increase the width of the $d_{0}$ distribution. A variation in the beam position results in an effective increase in beam size.

The lifetime is corrected for backgrounds present in the data sample, since the trimmed means of the backgrounds are different from those of real $\tau$ leptons. The resulting correction is $+1.01 \%$. The uncertainty on the correction is $0.24 \%$, and is assigned as a systematic error. An error also results from the uncertainty in the input Monte Carlo $\tau$ branching ratios, since the individual trimmed means of the different $\tau$ decay modes are also slightly different. This error is evaluated by eliminating one decay mode entirely from the Monte 
Table 1

Summary of the systematic error contributions to the one-prong $\tau$ lifetime measurement.

\begin{tabular}{ll}
\hline Source & Error \\
\hline resolution matching of Monte Carlo & $0.42 \%$ \\
detector calibration and alignment & $0.21 \%$ \\
beam size uncertainties & $0.33 \%$ \\
beam position uncertainties & $0.09 \%$ \\
background fractions & $0.24 \%$ \\
Monte Carlo decay mode branching ratios & $0.18 \%$ \\
Monte Carlo statistics & $0.39 \%$ \\
total systematic error & $0.76 \%$ \\
\hline
\end{tabular}

Carlo simulation and scaling the difference in lifetime by the relative uncertainty in the decay mode.

The systematic errors are summarized in Table 1. The total error is $0.76 \%$, and the correction is $+1.01 \%$. The final one-prong lifetime is $290.4 \pm 3.5 \pm 2.2 \mathrm{fs}$.

\section{Lifetime measurement using the decay length method}

In order to measure the $\tau$ lifetime using the decay length method, three-prong candidates are fitted to a vertex, which approximates the $\tau$ decay point, and the decay length is determined with respect to the beam position, which approximates the $\tau$ production point. A fit is performed to the decay length distribution to determine the average decay length, which is then converted to a lifetime using the boost factor, $1 / \beta \gamma c$.

In the determination of the decay length, threeprong candidates are required to have net charge \pm 1 and the track fit $\chi^{2}$ per degree of freedom of each track must be less than 10 . The three tracks are fitted to a vertex in the $r$ - $\phi$ plane, and the $\chi^{2}$ probability of the fit is required to be greater than 0.01 . To improve the precision with which the vertex is reconstructed, two of the three tracks must contain at least one silicon detector hit in the $r-\phi$ plane or have a majority of better resolution first hits from the axial wires of the vertex drift chamber [2]. Among several possible tracking selection requirements, this particular selection results in the highest efficiency while still providing reliable vertex reconstruction. The two-dimensional $(x-y)$ decay length and error are determined using a least squares fit combining the reconstructed beam position and error ellipse, the fitted vertex position and error ellipse, and using the event thrust axis as a direction constraint for the $\tau$ flight direction [2]. The resulting decay length and its error are converted into a three dimensional decay length using the polar angle of the thrust axis.

A four-parameter maximum likelihood fit is performed to the decay length distribution to determine the mean decay length. The fit function is a convolution of an exponential with the sum of two Gaussians. ${ }^{6}$ The four parameters in the fit are the mean decay length, $l_{0}$, two resolution scale factors for the decay length error, $s$ and $s_{2}$, and the fraction, $f_{2}$, of decay lengths which are described by the second scale factor. The two scale factors allow for a majority of well-measured vertices and the possibility of a small number of decays with larger errors. The fit is performed on the set of decay lengths whose errors are less than $0.6 \mathrm{~cm}$ and that lie within a window of $[-0.8,1.5] \mathrm{cm}$. A renormalization procedure is used to compensate for events removed by the decay length window cut [2]. All decay lengths used in the fit were boosted by the ratio of the reference centre-of-mass energy of $E_{\mathrm{cm}}=91.160 \mathrm{GeV}$ to their event $E_{\mathrm{cm}}$ (a small correction since all the data in 1994 were collected very near the $Z^{0}$ peak). There are 8696 vertices in the fit, and the resulting mean decay length is $0.2208 \pm 0.0028 \mathrm{~cm}$. The other fit parameters are $s=0.941 \pm 0.028, f_{2}=6.5 \pm 2.0 \%$ and $s_{2}=2.6 \pm 0.4$, where the errors shown are statistical only. A primary scale factor smaller than unity implies that the tracking errors are slightly overestimated, but does not have consequences for the lifetime measurement since the mean decay length and the resolution scale factor are almost uncorrelated.

The mean decay length is converted to a lifetime using a boost factor of $1301.4 \mathrm{fs} / \mathrm{cm}$, which includes the effects of initial state radiation, as determined in a Monte Carlo simulation assuming a $\tau$ mass of $1.777 \mathrm{GeV} / c^{2}$. The resulting raw lifetime is $287.3 \pm$ 3.6 fs.

The decay length distribution is shown in Fig. 2. Superimposed on this plot is a representation of the

\footnotetext{
${ }^{6}$ It is reasonable to assume an exponential form for the decay length distribution since at the $Z^{(1)}$ there is very little initial state radiation
} 


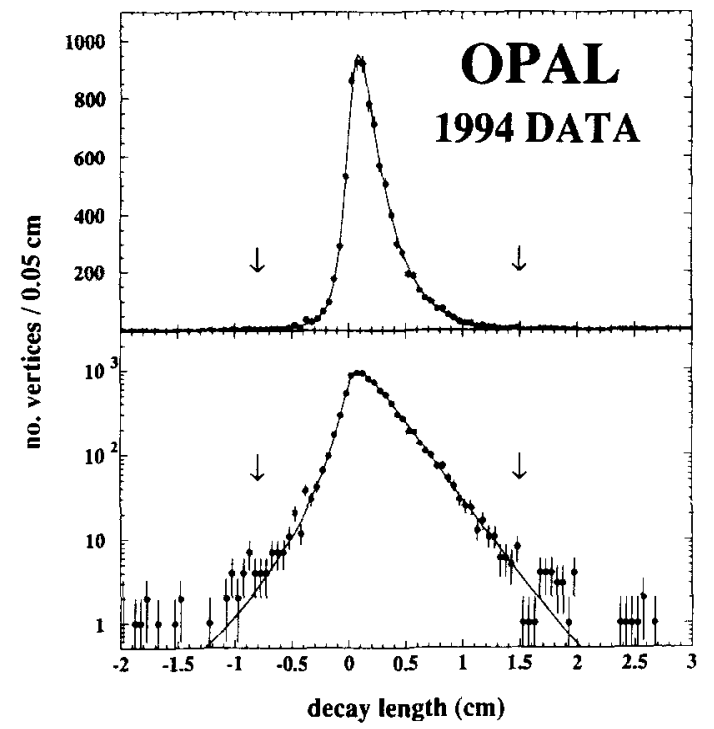

Fig. 2. The decay length distribution for selected vertices. The result of the decay length fit is shown as the smooth curve superimposed on the plot. The lifetime was determined within the range of decay lengths delimited by the arrows.

result of the maximum likelihood fit. A $\chi^{2}$ can be calculated by comparing this curve with the bin entries and their errors. By using only those bins within the fit range, the $\chi^{2}$ is 32.4 for 43 degrees of freedom, corrcsponding to a probability of $83 \%$.

The lifetime measurement is checked by determining it in bins of the polar and azimuthal angles of the thrust axis, the 3-prong charge, as well as for different choices for vertex probability cuts and decay length window cuts. The intrinsic spatial resolution of the silicon detector used in the track reconstruction is varied, as is the choice of thrust axis (three-prong momentum, combinations of charged tracks and electromagnetic calorimeter information). All the results are consistent.

Systematic errors in the decay length analysis are considered to come from six sources: the uncertainty in the radial position of the silicon detectors, the uncertainty in the drift velocity in the vertex chamber, beam position and beam size uncertainties, multihadronic background in the three-prong sample, and residual measurement biases as estimated from the Monte Carlo.

Alignment studies indicate that the radial position of the silicon detectors may be uncertain by as much as $50 \mu \mathrm{m}$ and this could introduce a systematic effect in the determination of decay lengths. The effect is modeled by shifting both layers of silicon detectors coherently and redetermining the decay length. However, because the selection of the three-prong candidates does not require every track to have silicon detector hits, the resulting systematic $(0.35 \%)$ is much smaller than one might naively expect (since $50 \mu \mathrm{m} / 2 \mathrm{~mm} \approx$ $2 \%)$.

The uncertainty in the drift velocity in the vertex chamber is less than $0.05 \%$. To determine the systematic crror contribution from this source, tracks without silicon detector hits are systematically shifted towards or away from the anode wire plane by amounts that simulate such an uncertainty.

The effects of the uncertainty in the beam size and position were studied by changing these parameters in the data. The sizes of the uncertainties in these parameters were given in Section 4. The beam size and position were varied separately in both $x$ and $y$, and the percentage changes in the decay length were combined in quadrature.

The fraction of background from multihadronic events remaining in the three-prong sample is $0.58 \%$, as described above, and the uncertainty in this background is $0.20 \%$. This small component of multihadronic background has been measured to have zero decay length and results in a $+0.58 \%$ correction to the lifetime.

Finally, there is a systematic error assigned for residual biases in the method. Although the Monte Carlo simulation is not used explicitly in this analysis, $j$ t is used to check whether there is a bias in the method, and the statistical error on the Monte Carlo simulation represents the precision with which this bias can be determined. The lifetime obtained from the Monte Carlo sample, following exactly the same procedure applied to the data, is consistent with the lifetime of the generated three-prong sample to within the level of the statistical error from the fit.

The systematic errors are summarized in Table 2. The total systematic error is $0.61 \%$, and the correction is $+0.58 \%$. The final lifetime from the decay length measurement is $289.0+3.6+1.8 \mathrm{fs}$. 
Table 2

Summary of the systematic error contributions to the three-prong $\tau$ lifetime measurement.

\begin{tabular}{ll}
\hline Source & Error \\
\hline silicon detector alignment & $0.35 \%$ \\
vertex chamber calibrations & $0.03 \%$ \\
beam size uncertainties & $0.06 \%$ \\
beam position uncertainties & $0.19 \%$ \\
multihadronic background & $0.20 \%$ \\
bias estimate & $0.41 \%$ \\
total systematic error & $0.61 \%$ \\
\hline
\end{tabular}

\section{Combination of results}

The results of this analysis are combined assuming that the detector calibration and alignment errors in the one-prong analysis are $100 \%$ correlated with the silicon detector alignment and vertex chamber calibration errors in the three-prong analysis. All other errors are assumed to be uncorrelated. The resulting lifetime is $\tau_{\tau}=289.7 \pm 2.5 \pm 1.5 \mathrm{fs}$.

Combined with the earlier measurements $[1,2]$, this yields a value for the $\tau$ lifetime based on the 19901994 data of $\tau_{\tau}=289.2 \pm 1.7 \pm 1.2$ fs. For this combination, correlations the same as those described above are assumed for one- and three-prong measurements from the same year. For the one-prong measurements from different years, the correlated quantities are the detector calibration and alignment, the background corrections, and the branching ratio errors. For the three-prong measurements from different years, the correlated quantities are the silicon detector alignment error, the vertex chamber calibration error, and the multihadronic background errors.

\section{Conclusions}

A precise measurement of the $\tau$ lepton lifetime has been made using $51007 \tau$ pair events collected using the OPAL detector in 1994, and has this been combined with earlier results to produce a single lifetime for all OPAL data from 1990-1994 of

$\tau_{\tau}=289.2 \pm 1.7$ (stat.) \pm 1.2 (syst.) fs,

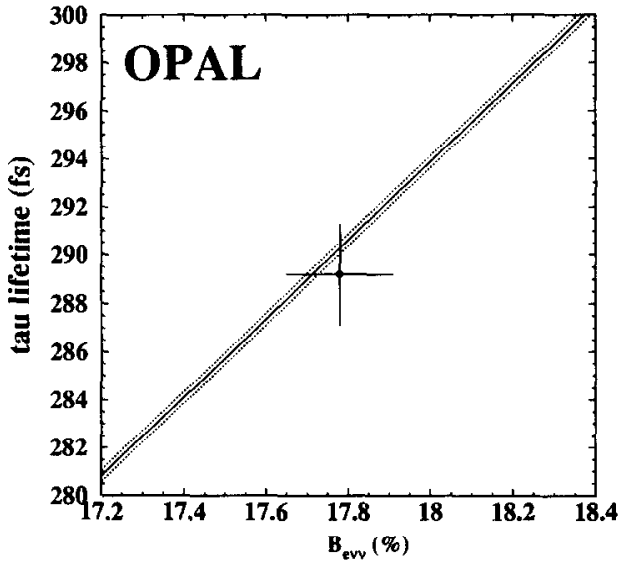

Fig. 3. The OPAL $\tau$ lifetime measurement is plotted against the OPAL measurement of the $\tau$ electronic branching ratio, $B_{e \nu \nu}$. The straight line shows the prediction assuming lepton universality, and the dotted lines relect the uncertainty associated with the $\tau$ mass.

which supersedes all previous OPAL results. This value is consistent with the recent lifetime measurements from ALEPH [14], DELPHI [15], SLD [16] and other experiments [17].

The new lifetime measurement can be used to check the $\tau$ - $\mu$ charged current lepton universality within the Standard Model. The form of this check was described in the introduction. The parameters used in the check are the precisely measured $\mu$ mass and lifetine [5] and the $\tau$ mass $\left(1776.96_{-0.21-0.17}^{+0.18+0.25} \mathrm{GeV} / c^{2}[4]\right)$. The remaining inputs, which currently limit the precision of the test, are the $\tau$ leptonic branching ratio and lifetime. Using the recent OPAL result for the electronic branching fraction of the $\tau$ lepton [18] of $\operatorname{BR}(\tau \rightarrow$ $\left.\mathrm{e} \bar{\nu}_{\mathrm{e}} \nu_{\tau}\right)=0.1778 \pm 0.0013$ and the above result for the $\tau$ lifetime, the ratio $g_{\tau} / g_{\mu}$ is $1.002 \pm 0.005$, consistent with the hypothesis of lepton universality. A comparison of the OPAL $\tau$ lifetime and electronic branching ratio measurements with the lepton universality prediction is shown in Fig. 3.

\section{Acknowledgements}

It is a pleasure to thank the SL Division for the efficient operation of the LEP accelerator, the precise information on the absolute energy, and their continuing close cooperation with our experimental group. In addition to the support staff at our own institutions 
we are pleased to acknowledge the Department of Energy, USA,

National Science Foundation, USA, Particle Physics and Astronomy Research Council, UK,

Natural Sciences and Engineering Research Council, Canada,

Israel Ministry of Science,

Israel Science Foundation, administered by the Israel Academy of Science and Humanities,

Minerva Gesellschaft,

Japanese Ministry of Education, Science and Culture (the Monbusho) and a grant under the Monbusho International Science Research Program,

German Israeli Bi-national Science Foundation (GIF),

Direction des Sciences de la Matière du Commissariat à l'Energie Atomique, France,

Bundesministerium für Bildung, Wissenschaft, Forschung und Technologie, Germany, National Research Council of Canada, Hungarian Foundation for Scientific Research, OTKA T-016660, and OTKA F-015089.

\section{References}

(1) OPAL Collab., R. Akers et al., Phys. Lett. B 338 (1994) 197.

[2] OPAL Collab., P.D. Acton et al., Z. Phys. C 59 (1993) 183

[3] W.J. Marciano and A. Sirlin, Phys. Rev. Lett. 61 (1988) 1815.

[4] BES Collab., J.Z. Bai et al., Phys. Rev. D 53 (1996) 20.

[5] Particle Data Group, L. Montanet et al., Phys. Rev. D 50 (1994) 1.

161 OPAL Collab., K. Ahmet et al, Nucl. Instrum. Meth. A 305 (1991) 275

[7] P.P. Allport et al., Nucl. Instrum. Meth. A 324 (1993) 34; A 346 (1994) 476.

[8| OPAL Cullab., G. Alexander el al., Z. Phys. C 52 (1991) 175.

19] S. Jadach, B.F.L. Ward and Z. Was, Comp. Phys. Comm. 79 (1994) 503 .

「10| J. Allison et al., Nucl. Instrum. Meth. A 317 (1992) 47.

[11] OPAL Collab., G. Alexander et al., Z. Phys. C 52 (1991) 175.

[12] T. Sjöstrand, Comp. Phys. Comm. 82 ( 1994) 74; JETSET 7.4 Manual, CERN-TH.7112/93.

[13] OPAL Collab., G. Alexander et al., CERN-PPE-95-126, to be published in $Z$. Phys. $C$.

[14] ALEPH Collab., D. Buskulic et al., CERN-PPE-95-128, submitted to Phys. Lett. B.

[15] DELPHI Collab., P. Abreu et al., CERN-PPE-95-154, submitted to Phys. Lett. B.

[16] SLD Collab., K. Abe et al., Phys. Rev. D 52 (1995) 4828.

17] Proceedings of the Third Workshop on Tau Lepton Physics, Nuclear Physics B (Proc. Suppl.) 40 (1995).

[18| OPAL Collab., G. Alexander et al., CERN-PPE-95-142, to be published in Phys. Lett. B. 